\title{
A REAL TIME MOTION CAPTURE SYSTEM, USING USB BASED TRI-AXIS MAGNETIC AND INERTIAL SENSORS, FOR MOVEMENT BASED RELAXATION.
}

\author{
John Foody ${ }^{\phi}$, Daniel Kelly ${ }^{\phi}$, Damini Kumar*, Diarmaid Fitzgerald*, Brian Caulfield*, \\ Tomas Ward $^{\phi}$ and Charles Markham ${ }^{\phi}$ \\ ${ }^{\phi}$ Department of Computer Science, \\ National University of Ireland, Maynooth \\ IRELAND \\ E-mail: jfoody@cs.nuim.ie \\ * School of Physiotherapy and Performance Science, \\ University College Dublin, \\ IRELAND \\ E-mail:*b.caulfield@ucd.ie
}

\begin{abstract}
A scalable motion capture system based on multiple orientation sensors, each consisting of tri-axis magnetic and gravitational field transducers, for use in kinematic studies; is presented. Outlined is a novel system capable of accessing real time data from multiple sensors, via one universal serial bus host, using a dynamic link library. In addition, design considerations; implementation and performance of the system are analysed and discussed.
\end{abstract}

Keywords - Motion Capture, Accelerometer, Magnetometer, Orientation Sensor, Yoga.

\section{INTRODUCTION}

In this project we are developing an effective feedback system for a human interface to promote mental and physical relaxation. Therapies such as Yoga and Tai Chi have been shown to have many therapeutic benefits in rehabilitation including enhancing postural awareness and reducing chronic pain and hypertension (Wu et al, 2004). We are designing a garment based physiological and kinematic measurement system in order to investigate these therapies. A system overview of which is shown in figure 1 . The system is integrated into a video game format. The entire garment is the human interface to a real-time kinematic feedback based video game, for promotion of yoga and Tai Chi.

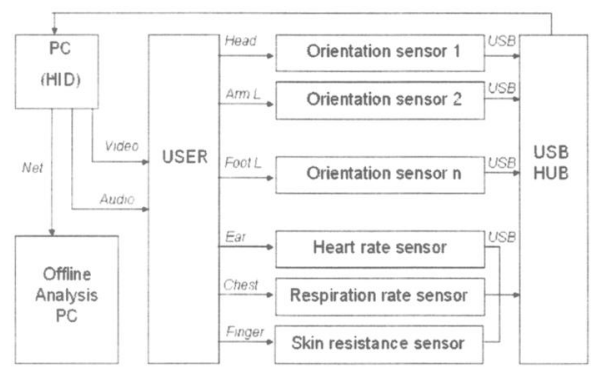

Figure 1: System Overview.
In order to achieve this, the development of an orientation sensor capable of scalable deployment was essential, multiples of which could be embedded on clothing worn by the subject at key kinematic tracking points on the body.

The sensor, an integrated tri-axis magnetic and inertial sensor package has been designed and implemented [1]; it is a Universal Serial Bus (USB) kinematic transducer, and therefore capable of scalable deployment. Moreover it is unobtrusive and because of its utilization of SMD technology has the ability to be miniaturized and realized on a small footprint. The orientation sensor developed is shown in figure 2 below:

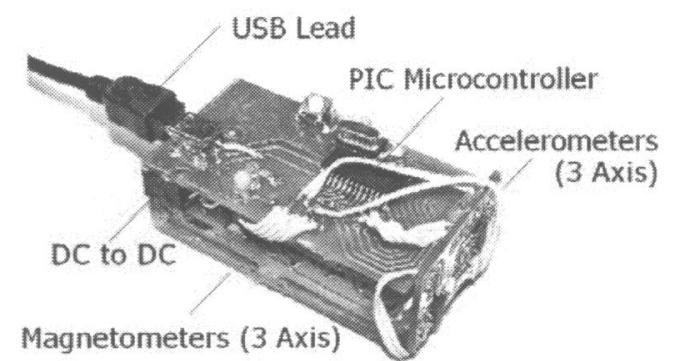

Figure 2: Prototype, USB Based Orientation Sensor. 
A software system to manage the introduction of multiple sensors was developed. In our specific application a minimum of nine orientation sensors are needed for full human motion tracking. A way to access data from anyone of these sensors at any time has been realized.

The sensor(s) are connected to the Host via a USB hub. Using a pre-compiled Dynamic Link Library (DLL), developed in the Visual Studio $6.0 \mathrm{C}++$, programming environment; a function call provides the user or software developer, with real-time access to kinematic data from any one of the connected sensors. The function call can be used in a series of programming environments, such as the now popular .NET framework.

\section{MATERIALS \& METHODS}

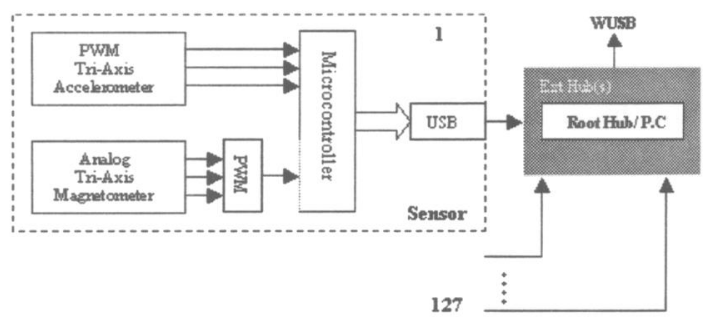

Figure 3: Block Diagram of Kinematic System

Each kinematic sensor, a block diagram of which can be observed in figure 3, contains a Microchip 16C745 Microcontroller, which along with some external circuitry provides the USB 1.1 client interface between the magnetic field and gravitational data, acquired from on board the sensor and the host software on the P.C.

Each sensor is programmed to act as a Human Interface Device, allowing use of the existing H.I.D drivers built in to MS Windows. The client code, residing on the Microcontroller, contains a USB standard product specific Vendor and product ID.

The host code, upon request accesses the USB host controller and looks for a Product and Vendor ID the matches its own. When a match is found a handle is returned, this handle is indexed in an array. This process continues until all devices have been identified.

Following this the host code populates a two dimensional array of size, $\mathrm{N}$ (where $\mathrm{N}$ is the number of handles returned) by six. It should be noted that the 16C745 Microcontroller returns six-pulse width modulated readings to the host code, corresponding to the three axis magnetic field and accelerometer readings, each with an equivalent resolution of 10 bits.
The host code represented as a series of function definitions built around the Windows Driver Development Kit and is structured as a Win32 DLL project in the Visual Studio C++ 6.0 programming environment. A .def file contains definitions required for the entry points for the relative functions within in the DLL.

The .dll and .def files can then be used with the sensors, by developers, to provide function calls, to return handles to all connected orientation sensors and to access the respective orientation data from them simultaneously and in real-time.

\section{EXPERIMENTS \& RESULTS}

In order to establish the functionality of the sensor a series of tests were performed, the outline and results of which are as follows:

Only results for one of the Euler Angles obtained by using accelerometers are provided (i.e. Pitch). In practice the six values produced by the sensor are put through a running average of twenty samples in order to reduce noise, this is before the values are normalised and worked on to provide standard Euler Angle values[1].

Initially the sensor was positioned stationary for 60 seconds; the yielded values for Yaw and Pitch and showed a standard deviation of 1.39 and 0.73 respectively [2].

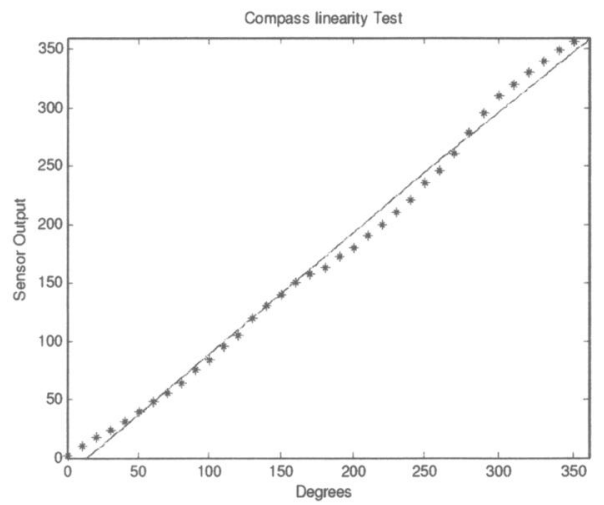

Figure 4: Yaw Linearity Test (Magnetometer)

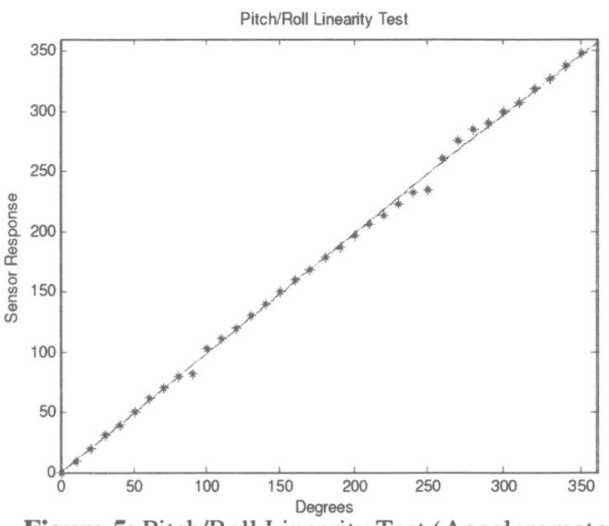

Figure 5: Pitch/Roll Linearity Test (Accelerometer) 


\begin{tabular}{||l|c|c||}
\hline & YAW & PITCH \\
\hline Slope & $1.036 \pm 0.02$ & $0.99 \pm 0.001$ \\
\hline Intercept & $-14.7 \pm 3.10$ & $-0.076 \pm 1.25$ \\
\hline \hline
\end{tabular}

Table 1: Linearity Test Results.

The sensor was then rotated in steps of ten degrees around the x-axis (pitch), and then around the z-axis (yaw) horizontal to the earths surface, meaning no tilt compensation was required and by extension not allowing any error on the part of pitch and or roll readings to affect the determination of yaw accuracy.

These results were then plotted (Figures 4 and 5) and the method of least squares was used to determine the slope and intercept of each [2] the results of which can be observed in Table 1 .

The current drawn by each sensor was recorded to be 170 milliamps.

Furthermore, in order to test and demonstrate the scalability of the system, three of the orientation sensors were used. The .dll and .ref file were integrated as part of a prototype, real-time kinematic feedback based video game. The game programmed using the .NET environment, avails of 'Direct $X$ ' to display a user controllable avatar on the screen.

All the joints of the avatar are defined by the BioVision Hierarchical (BVH) animation standard; the animation standard specification which only stores translation data for the root (the lowest point in the hierarchy) but requires rotational data in the form of Euler Angels for all joints [4]. For this game all of the avatar joints were pre-defined by a $\mathrm{BVH}$ file except the left arm.

The three, USB based orientation sensors were then connected through an active USB hub to the USB host on a P.C and positioned at key points on the left arm of a game player. The left arm of the subject was successfully replicated in real-time by the left arm of the games avatar.

In addition, data rates of up to twenty samples per second were recorded for all three sensors running simultaneously.

A screen shot of the prototype game application developed can be seen in Figure 6.

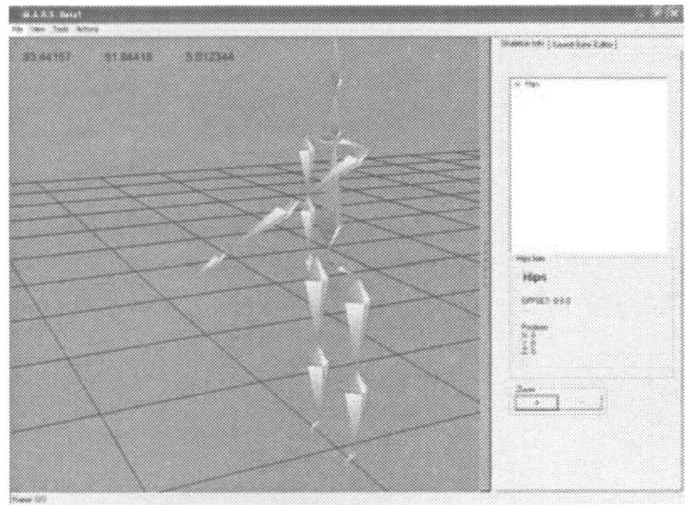

Figure 6: Screenshot of Prototype Game Developed.

\section{DISCUSSION}

The slope recorded for both Yaw and Pitch can be seen to give rise to a linear response in both experiments (figures 3-4).

However, the erroneous values produced as Pitch tends between 85-95 and 175-185 degrees can be attributed to a singularity error related to the mathematical approach taken in finding the Euler angles, alternative methods could be adopted to rectify this [1] [7].

The non-linearity's observed in Yaw and relatively large intercept error can be attributed to soft iron interference, this effect is dependant on the sensor angle, meaning the sensors magnetic field axes weren't precisely horizontal to the earth's surface when the measurements were taken. However, in practice this is compensated for by using the 3rd magnetic field vector[3], which is positioned perpendicular to the earth's surface, in collaboration with simultaneous measurement for pitch and roll i.e. electronic gimballed or tilt compensation [1]

The fact that the sensor currently draws 170 milliamps is significant as the USB specification [4] allows for a maximum of 500 milliamps to be made available to any one USB host. This means that when connecting more than two orientation sensors to a p.c and active hub is required [5].

It should be noted that, in relation to the USB host one hundred and twenty seven devices is the theoretical limit as long as enough current is available to power all these devices externally. Also, once the backward compatibility of the forthcoming wireless USB consumer products are utilised, a wireless human motion capture system can be achieved with ease. [6]

The system developed allows multiple orientation sensors to be interfaced simply with a P.C. In addition to this the API developed allows users to develop code for a range of specific applications. An example of one such application is outlined in this paper, the left arm tracking of a subject in real-time, represented by an on screen avatar.

\section{CONCLUSION}

This paper details the design and implementation of a scalable motion capture system based on multiple USB based sensors. Each sensor consists of tri-axis magnetic and gravitational field transducers. A system has been developed, capable of accessing real time data from three such sensors simultaneously via a single USB host and an active hub, with a signal command.

Finally, the system was tested and integrated in to a prototype application that provided the user with realtime visual feedback of their left arm moving. 


\section{ACKNOWLEDEMENTS}

The authors would like to thank the Higher Education Authority of Ireland for their support in funding this project and those involved at the National University of Ireland Maynooth and University College Dublin.

\section{REFERENCES}

[5] "Universal Serial Bus Revision 1.1

SPECIFICATION" www.usb.org/developers/docs;

Accessed: 09/12/04

[6]"WIRELESS USB, A WHITE PAPER"

http://www.intel.com/technology/comms/wusb

/download/wirelessUSB.pdf

Accessed: 11/09/05

(Books)

[2] BARFORD, N. C. (1969): 'Experimental Measurements: Precision, Error and Truth', (Addison-Wesley Publishing Company Inc.)

(Conference Proceedings)

[1] J.Foody D.Kumar D.Fitzgerald B.Caulfield T.Ward C.Markham, "A USB Interfaced Motion Capture Sensor, Using Tri-Axis Magnetic/Inertial Sensors For Use In Kinematic Studies." IEEE European conference on Biomedical Engineering. - Embec, Prague. November 2005.

(Technical Document)

[3] "Philips Semiconductors ApPlication NoteELECTRONIC COMPASS DESIGN USING KMZ51 AND KMZ52" -www.philips.semiconductors.com: Accessed:11/9/04

[4] M. Merdith and S.Maddock, "Motion File Formats Explained" http://www.dcs.shef.ac.uk/intranet/research/resmes/CS Accessesed: June 2005

(Journal publications)

[7] Ronf Zhu., and Ahaoying Zhu. (June 2004):

'A Real - Time Articulated Human Motion Tracking Using Tri-Axis Inertial/Magnetic Sensors Package', IEEE Transactions on Neural Systems and

Rehabilitation Engineering. 12, 2 